\title{
Epífora e flacidez sem ectrópio: "tarsal strip" resolve sempre?
}

\author{
Tearing and eyelid laxity with noectropion: Is tarsal strip always effective?
}

Denis Knijnik*

Trabalho realizado no Hospital Petrópolis - Porto Alegre (RS) - Brasil.

* Oftalmologista. Instrutor de Plástica Ocular do Instituto de Oftalmologia Prof. Ivo Corrêa-Meyer - Porto Alegre (RS) - Brasil.

Endereço para correspondência: Rua Cel. Bordini, 830 conj. 303 - Porto Alegre (RS) CEP 90440-003

E-mail: kdenis@ portoweb.com.br

Recebido para publicação em 10.08.2005 Aprovação em 27.10.2005

\begin{tabular}{|l|}
\hline RESUMO \\
\hline Objetivo: Verificar se o "tarsal strip" melhora a epífora de pacientes sem \\
ectrópio, mas com pálpebras flácidas. Métodos: Foi feito estudo retrospec- \\
tivo que incluiu uma amostra composta por pacientes com pálpebras \\
flácidas em posição normal, sem obstrução lacrimal, operados com \\
"tarsal strip" lateral para o tratamento de epífora. Foram incluídas no \\
estudo 14 pálpebras inferiores em 11 pacientes. Resultados: Houve \\
resolução ou melhora significativa da epífora em 10 (71,4\%) olhos após \\
período de seguimento de três meses. Todas as pálpebras operadas \\
passaram a apresentar resistência normal após a cirurgia. Conclusão: Em \\
pacientes com epífora, pálpebras flácidas e vias lacrimais pérvias, sem \\
ectrópio, o "tarsal strip" pode obter melhora significativa da epífora na \\
maioria dos casos. Como todas as pálpebras operadas recuperaram sua \\
resistência normal, pode-se supor que a ausência de melhora da epífora, \\
em alguns casos, possa ser explicada pela presença de outros fatores \\
causais concomitantes com a flacidez palpebral. \\
\hline
\end{tabular}

Descritores: Doenças do aparelho lacrimal/cirurgia; Pálpebras/cirurgia; Pálpebras/patologia; Técnicas de sutura; Idoso; Estudos retrospectivos

\section{INTRODUÇ̃̃̃O}

Muitos pacientes idosos queixam-se de lacrimejamento (epífora) apesar de não terem obstrução de vias lacrimais nem ectrópio de pálpebras inferiores $^{(1)}$. Menos evidente que ectrópio, como causa de epífora, é a pálpebra flácida que conserva sua posição normal, pois o seu diagnóstico depende de testar-se a distensibilidade da pálpebra.

Alterações de posição e elasticidade da pálpebra foram tratadas com técnicas cirúrgicas variadas ${ }^{(1-2)}$. Descrita em 1979 por Anderson $^{(3)}$, a técnica conhecida como "tarsal strip" vem sendo amplamente utilizada ${ }^{(4-5)}$. A técnica consiste em dissecar uma faixa do tarso e suturá-la ao periósteo do rebordo orbitário lateral. Em pacientes com epífora associada a ectrópio, o tarsal strip traz melhoras cosméticas e funcionais ${ }^{(6)}$.

O efeito do "tarsal strip" sobre a epífora de pacientes com pálpebras flácidas, mas sem ectrópio, foi avaliado retrospectivamente por Vick et al ${ }^{(7)}$. Nosso objetivo foi verificar, de forma semelhante, o efeito do "tarsal strip" especificamente sobre a epífora de pacientes com pálpebras flácidas em posição normal e sem obstrução lacrimal.

\section{MÉTODOS}

Este estudo retrospectivo incluiu pacientes com queixa de epífora, que apresentavam flacidez palpebral sem ectrópio, operados com "tarsal strip" 
lateral e que atenderam a todos os critérios seguintes: flacidez de pálpebra inferior; ausência de ectrópio ou entrópio; pontos lacrimais com aspecto e posição normais; retenção de contraste verificada no teste do desaparecimento da fluoresceína; perviedade do sistema lacrimal demonstrada à dacriocistografia, sondagem ou irrigação.

A flacidez era diagnosticada examinando-se a distensibilidade e resistência da pálpebra, tanto no sentido inferior quanto horizontal, e a rapidez no retorno à posição inicial (snapback). Pacientes em quem, ao puxar-se a pálpebra lateralmente, verificava-se que o componente medial da flacidez era predominante, não fizeram parte do estudo.

Para afastar outras variáveis, foram incluídos somente os casos em que, por ocasião da cirurgia, não foram realizados outros procedimentos, como punctoplastia, sondagem ou irrigação.

Foram incluídas no estudo 14 pálpebras inferiores operadas com "tarsal strip" lateral em 11 pacientes. As cirurgias foram realizadas no período de agosto de 2000 a novembro de 2004. A idade dos pacientes variou de 59 a 84 anos (média: 70,8 anos). A amostra incluiu somente pacientes que retornaram para avaliação três meses após a cirurgia.

O trabalho foi aprovado pelo Comitê de Ética da instituição.

A execução do "tarsal strip"(3) incluiu os seguintes passos básicos: cantotomia lateral de $1 \mathrm{~cm}$; cantólise lateral (tendão cantal lateral é incisado de modo a liberar completamente a pálpebra da órbita); incisão na linha cinzenta da pálpebra de modo a separar a lamela anterior da posterior (extensão da incisão depende da quantidade necessária de encurtamento da pálpebra). A parte medial desta incisão demarca a nova comissura lateral da pálpebra. Uma faixa de tarso (tarsal strip) é preparada e, depois, suturada ao periósteo da face interna do rebordo orbitário lateral. A comissura lateral é reconstituída.

\section{RESULTADOS}

Houve resolução ou melhora significativa da epífora em 10 $(71,4 \%)$ olhos após um período de seguimento de três meses. $\mathrm{O}$ critério de melhora foi a informação do paciente de que a epífora havia cessado inteiramente ou quase.

A totalidade das pálpebras operadas passou a apresentar resistência normal após a cirurgia.

\section{DISCUSSÃO}

Enquanto irritação ocular externa crônica é parte integrante das situações denominadas como "floppy eyelid syndrome"(8) e "lax eyelid syndrome"(9-10), a flacidez das pálpebras inferiores pode causar simplesmente disfunção da bomba lacrimal e epífora.

A epífora atribuída à flacidez palpebral foi estudada há quase trinta anos por Hurwitz ${ }^{(1)}$, cuja amostra, apesar de excluir casos de ectrópio declarado, incluía também pacientes operados por eversão e estenose de ponto lacrimal e flacidez medial da pálpebra. As técnicas cirúrgicas foram variadas e escolhidas de acordo com a alteração encontrada, tendo obtido bons resultados.

Limitando sua amostra aos casos sem ectrópio nem alterações de ponto lacrimal, Vick et al.(7) obtiveram ótimas respostas quanto à resolução da epífora utilizando o "tarsal strip".

O nosso estudo utilizou uma amostra cujos critérios de exclusão e inclusão foram semelhantes aos de Vick et al. Apesar de todos os nossos casos terem tido a distensibilidade palpebral corrigida com sucesso, nossos resultados quanto à epífora foram um pouco inferiores aos deles.

Cabe indagar a origem dos insucessos, especialmente tendo-se em vista que todas as pálpebras operadas passaram a ter elasticidade normal após o "tarsal strip".

A ausência de melhora poderia ser atribuída à coexistência da flacidez palpebral com algum outro fator. Como a flacidez de pálpebras inferiores é freqüentemente identificada em pacientes que não têm epífora, pode ser necessária a existência concomitante de mais de um fator causal para que ocorra epífora. Um desses fatores associados pode ser uma obstrução parcial ou funcional do canal nasolacrimal. Em comum, ambas as patologias produzem retenção no teste de desaparecimento da fluoresceína. Se bem que em ambas ocorre passagem de contraste para a fossa nasal, a dacriocistografia pode revelar alterações que contribuem para o diagnóstico de obstrução parcial, como dilatações do saco lacrimal e estreitamentos do canal. Para tratar a obstrução parcial, a dacriocistorrinostomia endonasal é especialmente útil ${ }^{(11)}$.

Os resultados basearam-se na informação prestada pelo paciente. $\mathrm{O}$ relato pelo próprio paciente de que a epífora desapareceu é o alvo a ser alcançado com o tratamento, constituindo-se no melhor critério de sucesso. Testes como retenção de fluoresceína ou Jones, no pós-operatório, podem sabidamente dar respostas em desacordo com o resultado experimentado pelo paciente.

Embora tenhamos avaliado subjetivamente a distensibilidade da pálpebra, esta medida na prática é suficiente e de uso corrente entre os especialistas. Ainda não há um sistema padronizado amplamente aceito de quantificação, apesar das contribuições nesse sentido ${ }^{(12-13)}$.

\section{CONCLUSÃO}

Em pacientes com epífora, pálpebras flácidas e vias lacrimais pérvias, sem ectrópio, o "tarsal strip" lateral pode obter melhora significativa da epífora na maioria dos casos.

Embora a totalidade dos olhos operados tenha recuperado a distensibilidade normal da pálpebra inferior após o "tarsal strip", nem todos tiveram melhora da epífora. Isso permite supor que possa haver outros fatores causais, simultâneos à flacidez palpebral, que necessitam ser identificados e tratados. 


\section{ABSTRACT}

Purpose: To verify whether the tarsal strip procedure improves epiphora of patients with eyelid laxity but no ectropion. Methods: A retrospective study was done with a sample of epiphora patients with lax eyelids in normal position and no nasolacrimal obstruction who were operated on with the tarsal strip technique. Fourteen lower eyelids, in 11 patients, were included in the study. Results: Epiphora in ten eyes $(71.4 \%)$ completely or significantly improved after a follow-up of three months. Eyelid laxity disappeared in all cases. Conclusion: In patients with epiphora, lax eyelids and patent lacrimal ducts, the tarsal strip procedure can significantly improve epiphora in most cases. As laxity ceased to exist in all eyelids, an assumption can be made that in some of the failures there may be other causes, concomitant with eyelid laxity, to explain perseverance of epiphora.

Keywords: Lacrimal apparatus diseases/surgery; Eyelids/ surgery; Eyelids/pathology; Suture techniques; Retrospective studies; Aged

\section{REFERÊNCIAS}

1. Hurwitz JJ. Investigation and treatment of epiphora due to lid laxity. Trans Ophthalmol Soc U K. 1978; 98(1):69-70.

2. Hurwitz JJ, Mishkin SK, Rodgers KJ. Modification of Bick's procedure for treatment of eyelid laxity. Can J Ophthalmol. 1987;22(5):262-5.

3. Anderson RL, Gordy DD. The tarsal strip procedure. Arch Ophthalmol. 1979; 97(11):2192-6.

4. Lessa S, Sebastiá R, Pitanguy I. Retalho tarsal. Rev Bras Cir. 1989;79(3): 175-9.

5. Sbrissa RA, Sbrissa Júnior RA. Ectrópio senil - correção pela técnica da fita tarsal. Rev Bras Oftalmol. 1993;52(3):41-3.

6. Kielhorn I, Rowson NJ. Lateral canthal surgery in the management of epiphora. Orbit. 2002;21(2):111-6.

7. Vick VL, Holds JB, Hartstein ME, Massry GG. Tarsal strip procedure for the correction of tearing. Ophthal Plast Reconstr Surg. 2004;20(1):37-9.

8. Culbertson WW, Ostler HB. The floppy eyelid syndrome. Am J Ophthalmol. 1981;92(4):568-75.

9. van den Bosch WA, Lemij HG. The lax eyelid syndrome. Br J Ophthalmol. 1994;78(9):666-70

10. Burkat CN, Lemke BN. Acquired lax eyelid syndrome: an unrecognized cause of the chronically irritated eye. Ophthal Plast Reconstr Surg. 2005;21(1):52-8.

11. Knijnik D, Uebel VN, Santos RS. Dacriocistorrinostomia endoscópica endonasal em casos de epífora com vias lacrimais pérvias. Arq Bras Oftalmol. 2003;66(2):173-5.

12. Olver JM, Sathia PJ, Wright M. Lower eyelid medial canthal tendon laxity grading: an interobserver study of normal subjects. Ophthalmology. 2001; 108(12):2321-5

13. Francis IC. RE: "Canthus-sparing ectropion repair". Ophthal Plast Reconstr Surg. 2002;18(4):310-1; author reply 311-2

\section{Simpósio de Córnea e Glaucoma do Hospital Oftalmológico de Sorocaba}

\section{0 e 21 de Outubro de 2006 SOROCABA - SP}

\section{INFORMAÇÕES}

Tel.: (15) 3212-7077 • E-mail: ceo@hosbos.com.br 